\title{
Sperm DNA fragmentation
}

It is an honour to write the foreword to this focused issue of TAU on sperm DNA fragmentation (SDF). From my time as a first generation embryologist in the early days at the University of Cambridge with Professor Robert Edwards and in my current role at the London Women's Clinic, I have become acutely aware of the number of practicalities that still need to be solved in the field of IVF and assisted reproduction. Whilst gene technology and ability to perform mitochondrial transfer are the latest advances applied to early embryos, there are other significant developments, much closer to home, that can influence more people to achieve their goal of achieving a healthy family.

Our increasing awareness of DNA damage in the male germ line and its effect on reproductive outcomes has been steadily improving. Measuring SDF has been shown to differentiate between infertile and fertile males with higher levels of SDF correlating with decreased fertility. A wealth of studies demonstrate that SDF can effect fertilisation, implantation, risk of miscarriage and slow early embryo development. Hence, SDF is now an area of significance as evidenced by the vast array of the focused contributors from different countries around the world and the array of topics highlighted in this special issue.

Such high calibre research is essential to understand the mechanisms that cause SDF and the level of SDF that correlates with pregnancy outcome. One of the factors that contributes to SDF is oxidative stress; an area of research that has profoundly benefited from publications of the highest calibre from Ashok Agarwal and his team based at the Cleveland Clinic in Ohio. It is fitting that this team leads to provide such a comprehensive account of affairs on SDF in this special supplement.

From a clinical perspective, one of the challenges now is the best application of our knowledge of SDF in a commercial setting. When we consider donor banking for example, should we test every donor that we recruit to identify potent donors with very little SDF? If we do so should this be at the risk of reducing acceptance rates?

At a time when the value of 'add ons' are a highly debated topic in the field of fertility treatment, it is prudent to ensure that SDF tests have a robust predictive indicator of pregnancy success rates and also that they represent good value for money.

With these thoughts in mind I would like to welcome what looks to be a very interesting and powerful documentation on SDF for practitioners worldwide.

\section{Acknowledgements}

None. 


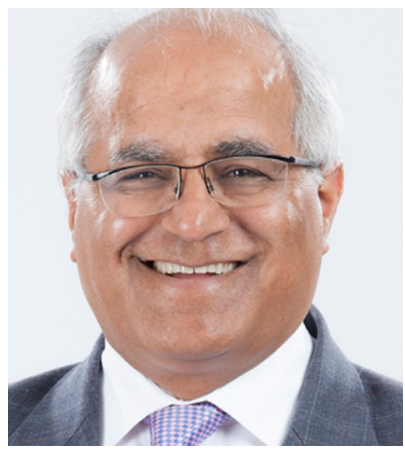

Kamal K. Ahuja

Kamal K. Ahuja, PhD Scientific and Managing Director, London Women's Clinic, London, UK; Chairman, Reproductive Biomedicine Online, Cambridge, UK.

(Email: Kamal.abuja@londonwomensclinic.com) doi: $10.21037 /$ tau.2017.08.11

Conflicts of Interest: The author has no conflicts of interest to declare.

View this article at: http://dx.doi.org/10.21037/tau.2017.08.11

Cite this article as: Ahuja KK. Sperm DNA fragmentation. Transl Androl Urol 2017;6(Suppl 4):S337-S338. doi: 10.21037/ tau.2017.08.11 